\title{
INEQUALITIES FOR WALSH LIKE RANDOM VARIABLES
}

\section{HAJELA}

Be11 Communications Research, 2P-390

445 South Street

Morristown, New Jersey, 07960, U.S.A.

(Received March 28, 1988)

ABSTRACT. Let $\left(X_{n}\right)_{n>1}$ be a sequence of mean zero independent random variables.

Let $\left.W_{k}={\underset{j=1}{k}}_{j=1}^{k} x_{1} \mid 1<i_{1}<1_{2} \ldots<i_{k}\right\}, Y_{k}=\bigcup_{j \& k} w_{j}$ and let $\left[Y_{k}\right]$ be the linear span of $\mathrm{Y}_{\mathrm{k}}$. Assume $\delta<\left|\mathrm{X}_{\mathrm{n}}\right|<\mathrm{K}$ for some $\delta>0$ and $\mathrm{K}>0$ and let

$C(p, m)=16\left(5 \sqrt{2} \frac{p^{2}}{p-1}\right)^{m-1} \frac{p}{\log p}\left(\frac{K}{\delta}\right)^{m}$ for $1<p<\infty$. We show that for $f \varepsilon\left[Y_{m}\right]$ the following inequalities hold:

$$
\begin{array}{ll}
\|\mathrm{f}\|_{2}<\|\mathrm{f}\|_{\mathrm{p}}<\mathrm{c}(\mathrm{p}, \mathrm{m})\|\mathrm{f}\|_{2} \quad \text { for } 2<\mathrm{p}<\infty \\
\|\mathrm{f}\|_{2}<\mathrm{c}(\mathrm{q}, \mathrm{m})\|\mathrm{f}\|_{\mathrm{p}}<\mathrm{c}(\mathrm{q}, \mathrm{m})\|\mathrm{f}\|_{2} & \text { for } 1<\mathrm{p}<2, \frac{1}{\mathrm{p}}+\frac{1}{\mathrm{q}}=1
\end{array}
$$

and $\|\mathrm{f}\|_{2}<\mathrm{C}(4, \mathrm{~m})^{2}|| \mathrm{f}\left\|_{1}<\mathrm{C}(4, \mathrm{~m})^{2}\right\| \mathrm{f} \|_{2^{\circ}} \quad$ These generalize various well known inequalities on Walsh functions.

KEY WORDS AND PHRASES. Walsh Functions, Martingales, Square Function. 1980 AMS SUBJECT CLASSIFICATION CODE. Primary 60E15, Secondary 60A99.

1. INTRODUCTION.

Let $\left(x_{n}\right)_{n>1}$ be a sequence of independent mean zero random variables. Let $W_{k}$ be products of length $k$ of the $\left(x_{n}\right)_{n>1}$ i.e.

$$
w_{k}=\left\{x_{1_{2}} x_{i_{2}} \ldots x_{1_{k}} \mid 1_{1}<1_{2}<\ldots<1_{k}\right\} \text {, }
$$

let $Y_{k}=\bigcup_{j \notin k} W_{j}$ and let $\left[Y_{k}\right]$ be the linear span of functions in $Y_{k}$. The object of this note is to show that for functions in $\left[Y_{k}\right]$ the $p^{\prime}$ th mean is of the same order as the second moment. As such this generalizes classical inequalities such as Khinchin's Inequality in Zygmund [1] as well as more recent inequalities on Walsh functions such as those of $\mathrm{H}$. Rosenthal [2] and A. Bonami [3]. Precisely we prove the following:

THEOREM 2.4. Let $\left(X_{n}\right)_{n}>1$ be a sequence of independent mean zero random variables on a probability space $(\Omega, \mu)$. Suppose there exist $\delta>0$ and $K>0$ such that $\delta<\left|X_{n}\right|<\mathrm{K}$ for all $\mathrm{n}$. For $\mathrm{f} \varepsilon\left[\mathrm{Y}_{\mathrm{n}}\right]$ we have 


$$
\begin{aligned}
& \left\|f f_{2}<\right\| f\left\|_{p}<c(p, n)\right\| f \|_{2} \text {, for } 2<p<\infty \\
& \|f\|_{2}<c(q, n)\|f\|_{p}<c(q, n)\|f\|_{2} \text {, for } 1<p<2, \frac{1}{p}+\frac{1}{q}=1 . \\
& \|f\|_{2}<c(4, n)^{2}\|f\|_{1}<c(4, n)^{2}\|f\|_{2}
\end{aligned}
$$

where $c(p, n)=16\left(5 \sqrt{2} \frac{p^{2}}{p-1}\right)^{n-1} \frac{p}{\log p}\left(\frac{k}{\delta}\right)^{n}$.

We assume of course that the $\left(X_{n}\right)_{n>1}$ belong to some $L^{p}(\Omega)$. Recall that for $1<\mathrm{p}<\infty, \mathrm{L}^{\mathrm{P}}(\Omega)$ is the space of all measurable functions $f$ such that $\int|f(\omega)|^{p} d_{\mu}<\infty$ and the norm of $f$ is $\|\left. f\right|_{p}=\left(\int|f(\omega)|^{\left.P_{d}\right)^{1 / p}}\right.$. We assume the reader is familiar with martingales and refer to Garsia [2] for unexplained notation.

2. PROOF OF THE INEQUALITIES.

We require three preliminary facts in order to prove theorem 2.4. We denote by $E(X)$, the expectation of a random variable $X$.

THEOREM 2.1. [1]. Let $r_{n}(t)$ be the Rademacher functions on $[0,1]$.

Then $\int_{0}^{1}\left|\sum_{k=0} a_{k} r_{k}(t)\right| d t>\frac{1}{\sqrt{2}}\left(\sum_{k}\left|a_{k}\right|^{2}\right)^{1 / 2}$ for any complex numbers $\left(a_{k}\right)_{k=1}^{n} \subseteq C$.

THEOREM 2.2. (Johnson, Schechtman, and $Z$ inn [5]) Let $\left(x_{n}\right)_{n>1}$ be a sequence of independent mean zero variables and let $\left(a_{k}\right)_{k=1}^{n} \varepsilon c^{n}$. Then for $p>2$

$$
\left\|\sum_{k=n} a_{k} x_{k}\right\|_{p}<\frac{16 p}{\log p} \max \left(\left\|\sum_{k \infty n} a_{k} x_{k}\right\|_{2},\left(\sum_{k \infty n}\left|a_{k}\right|^{p} E\left|x_{k}\right|^{p}\right)^{1 / p}\right) .
$$

Recall that for a martingale $f=\left(f_{n}\right)_{n>1}$, its difference sequence is $d_{n}=f_{n}$ $f_{n-1}$ and its square function is $S(f)=\left(\sum_{n} d_{n}^{2}\right)^{1 / 2}$. The last fact that we need is:

THEOREM 2.3 [4]. For a martingale $f=\left(f_{n}\right)$, we have

$$
\left\|f_{n}\right\|_{p}<\frac{5 p^{2}}{p-1}\left\|\left(\sum_{k \infty n} d_{k}^{2}\right)^{1 / 2}\right\|_{p} \text { for } 1<p<\infty .
$$

We may now prove Theorem 2.4 quite easily.

THEOREM 2.4. Let $\left(X_{n}\right)_{n>1}$ be a sequence of independent mean zero random var1ables on a probability space $(\Omega, \mu)$. Suppose there exist $\delta>0$ and $K>0$ such that

$\delta<\left|x_{n}\right|<K$ for all $n$. For $f \in\left[Y_{m}\right]$ we have

$$
\begin{aligned}
& \|\mathrm{f}\|_{2}<\|\mathrm{f}\|_{\mathrm{p}}<\mathrm{c}(\mathrm{p}, \mathrm{m})\|\mathrm{f}\|_{2} \text { for } 2<\mathrm{p}<\infty \\
& \|\mathrm{f}\|_{2}<\mathrm{c}(\mathrm{q}, \mathrm{m})\|\mathrm{f}\|_{\mathrm{p}}<\mathrm{c}(\mathrm{q}, \mathrm{m})\|\mathrm{f}\|_{2} \text { for } 1<\mathrm{p}<2
\end{aligned}
$$

and $1 / p+1 / q=1$.

$$
\|\mathrm{f}\|_{2}<\mathrm{c}(4, \mathrm{~m})^{2}\|\mathrm{f}\|_{1}<\mathrm{c}(4, \mathrm{~m})^{2}\|\mathrm{f}\|_{2}
$$


where $C(p, m)=16\left(5 \sqrt{2} \frac{p^{2}}{p-1}\right)^{m-1} \frac{p}{\log p}\left(\frac{k}{\delta}\right)^{m}$.

PROOF. The proof is by induction on $m$. We will first consider the case $p>2$. Suppose $m=1$ and $f \in\left[Y_{1}\right]$. Then $f=\sum_{k \in n} a_{k} X_{k}$ for some $a_{k} \varepsilon C, k=1, \ldots, n$. By Theorem 2.2 we have,

$$
\begin{aligned}
& \|f\|_{p}=\left\|\sum_{k \in n} a_{k} x_{k}\right\|_{p}<16 \frac{p}{\log p} \max \left(\left\|\sum_{k \in n} a_{k} x_{k}\right\|_{2},\left(\sum_{k \infty n}\left|a_{k}\right|^{p}\left|x_{k}\right|^{p}\right)^{1 / p}\right) \\
& =\frac{16 p}{\log p} \max \left(\left(\sum\left|a_{k}\right|^{2} E\left|X_{k}\right|^{2}\right)^{1 / 2},\left(\sum\left|a_{k}\right|^{P_{E}}\left|X_{k}\right|^{P}\right)^{1 / p}\right) \\
& \text { (Since } \mathrm{EX}_{\mathrm{k}}=0 \text { and the } \mathrm{X}_{\mathrm{k}} \text { are independent) } \\
& <\frac{16 \mathrm{pK}}{\log \mathrm{pax}}\left(\left(\sum_{\mathrm{q}}\left|\mathrm{a}_{k}\right|^{2}\right)^{1 / 2},\left(\sum_{k}\left|a_{k}\right|^{\mathrm{p}}\right)^{1 / \mathrm{p}}\right) \\
& =\frac{16 \mathrm{pK}}{\log p}\left(\sum_{k \in \mathrm{n}}\left|\mathrm{a}_{\mathrm{k}}\right|^{2}\right)^{1 / 2} .
\end{aligned}
$$
However $\|f\|_{2}=\left(\sum_{k \& n}\left|a_{k}\right|^{2} E\left|x_{k}\right|^{2}\right)^{1 / 2}>\&\left(\sum_{k \& n}\left|a_{k}\right|^{2}\right)^{1 / 2}$ and so by (2.4) the result
follows for $m=1$.

We assume the result is valid for $f \varepsilon\left[\mathrm{Y}_{\mathrm{m}}\right]$. Let $\mathrm{f} \varepsilon\left[\mathrm{Y}_{\mathrm{m}+1}\right]$. Note that we may write $f$ as $f=\sum_{n>1} f_{n} X_{n}$ where $f_{n} \varepsilon\left[Y_{m}\right]$ and $f_{n}$ only depends on the random variables

$x_{j}, 1<j<n$. It is clear then that $f$ is a sum of a martingale difference sequence. Applying Theorem 2.3 we have

$$
\begin{aligned}
& \|f\|_{p}<5 \frac{p^{2}}{p-1}\|s(f)\|_{p} \\
& =5 \frac{p^{2}}{p-1}\left\|\left(\sum_{n>1} f_{n}^{2} x_{n}^{2}\right)^{1 / 2}\right\|_{p} \\
& \text { < } \left.5 \frac{\mathrm{p}^{2}}{\mathrm{p}-1} \mathrm{k}||\left(\sum_{\mathrm{n} \geqslant 1} \mathrm{f}_{\mathrm{n}}^{2}\right)^{1 / 2} \|_{\mathrm{p}} \text { (since }\left|\mathrm{x}_{\mathrm{n}}\right|<\mathrm{K}\right) \\
& <5 \frac{\sqrt{2} p^{2}}{p-1} k\left\|\int_{0}^{1}\left|\sum_{n>1} r_{n}(t) f_{n}\right| d t\right\|_{p} \text { (by Theorem 2.1) } \\
& <5 \sqrt{2} \frac{p^{2}}{p-1} k \int_{0}^{1}\left\|\sum_{k>1} r_{n}(t) f_{n}\right\|_{p} d t \\
& <5 \sqrt{2} \frac{\mathrm{p}^{2}}{\mathrm{p}-1} \mathrm{k}(\mathrm{p}, \mathrm{m}) \int_{0}^{1}\left\|\sum_{\mathrm{n}>1} \mathrm{r}_{\mathrm{n}}(\mathrm{t}) \mathrm{f}_{\mathrm{n}}\right\|_{2} \mathrm{dt} \text { (by induction) } \\
& <5 \sqrt{2} \frac{p^{2}}{p-1} K c(p, m)\left(\int_{0}^{1}\left\|\sum_{n \geqslant 1} r_{n}(t) f_{n}\right\|_{2}^{2}\right)^{1 / 2} \\
& =5 \sqrt{2} \frac{p^{2}}{p-1} K C(p, m)\left(\int_{0}^{1} \int_{\Omega}\left|\sum_{n \geqslant 1} r_{n}(t) f_{n}(\omega)\right|^{2} d_{\mu} d t\right)^{1 / 2} \\
& =5 \sqrt{2} \frac{p^{2}}{p-1} K C(p, m)\left(\iint_{\Omega}^{1}\left|\sum_{n \geqslant 1} r_{n}(t) f_{n}(w)\right|^{2} d t d \mu\right)^{1 / 2} \text { (by Fubini's } \\
& =5 \sqrt{2} \frac{p^{2}}{p-1} K C(p, m)\left(\int_{\Omega} \sum_{n \geqslant 1} f_{n}^{2}(\Omega) d \mu\right)^{1 / 2} \text { (since the Rademacher's are }
\end{aligned}
$$




$$
\begin{aligned}
& <5 \sqrt{2} \frac{\mathrm{p}^{2}}{\mathrm{p}-1} \frac{\mathrm{K}}{\delta} \mathrm{C}(\mathrm{p}, \mathrm{m})\left(\int_{\Omega \mathrm{n} \geqslant 1} \sum_{\mathrm{n}} \mathrm{f}^{2}(\mathrm{~s}) \mathrm{x}_{n}^{2}(\mathrm{u}) \mathrm{d} \mu\right)^{1 / 2}\left(\text { since }\left|\mathrm{x}_{\mathrm{n}}\right|>\delta\right) \\
& =5 \sqrt{2} \frac{\mathrm{p}^{2}}{\mathrm{p}-1} \frac{\mathrm{K}}{\delta} \mathrm{C}(\mathrm{p}, \mathrm{m})|| \mathrm{S}(\mathrm{f}) \|_{\mathrm{f}} \\
& =5 \sqrt{2} \frac{\mathrm{p}^{2}}{\mathrm{p}-1} \frac{\mathrm{K}}{\delta} \mathrm{C}(\mathrm{p}, \mathrm{m})|| \mathrm{f} \|_{2} \text { (since } \mathrm{f} \text { is a martingle) } \\
& =\mathrm{c}(\mathrm{p}, \mathrm{m}+1)|| \mathrm{f} \|_{2}
\end{aligned}
$$

Hence $\|\mathrm{f}\|_{\mathrm{p}}<\mathrm{C}(\mathrm{p}, \mathrm{m}+1)\|\mathrm{f}\|_{2}$ for $\mathrm{f} \varepsilon\left[\mathrm{Y}_{\mathrm{m}+1}\right]$ proving the result for $\mathrm{p}>2$. For $1<p<2$ we employ the classical trick of Holder. Let $q$ be defined by $\frac{1}{p}+\frac{1}{q}=1$. Then $\frac{1}{2}=\frac{1-\theta}{q}+\frac{\theta}{p}$ for $\theta=\frac{1}{2}$. Let $f \in\left[Y_{m}\right]$.

Then, $\|\mathrm{f}\|_{2}<\|\mathrm{f}\|_{\mathrm{q}}^{1 / 2}\|\mathrm{f}\|_{\mathrm{p}}^{1 / 2} \quad$ (by Hölder's Inequality)

$$
<\mathrm{C}(\mathrm{q}, \mathrm{m})^{1 / 2}|| \mathrm{f}\left\|_{2}^{1 / 2}\right\| \mathrm{f} \|_{\mathrm{p}}^{1 / 2}(\text { since } \mathrm{q}>2) \text {. }
$$

So $\|\mathrm{f}\|_{2}<\mathrm{C}(\mathrm{q}, \mathrm{m})\|\mathrm{f}\|_{\mathrm{p}}$ while $\|\mathrm{f}\|_{\mathrm{p}}<\|\mathrm{f}\|_{2}$ is obvious, proving (2.2). Finally to see (2.3) note that $\frac{1}{2}=\frac{1-\theta}{4}+\frac{\theta}{1}$ for $\theta=1 / 3$, so again by Ho'lder's inequality,

$$
\left.\|\mathrm{f}\|_{2}<\|\mathrm{f}\|_{4}^{2 / 3}|| \mathrm{f}\left\|_{1}^{1 / 3}<\right\| \mathrm{f}\left\|_{2}^{2 / 3} \mathrm{c}(4, \mathrm{~m})^{2 / 3}\right\| \mathrm{f} \|_{1}^{1 / 3} \text { (for } \mathrm{f} \varepsilon\left[\mathrm{Y}_{\mathrm{m}}\right]\right) \text {. }
$$

So $\|\mathrm{f}\|_{2}<\mathrm{c}(4, \mathrm{~m})^{2}|| \mathrm{f} \|_{1}$, while $\|\mathrm{f}\|_{2}>\|\mathrm{f}\|_{1}$ 1s automat1c, proving (2.3).

\section{REFERENCES}

1. ZYGMUND, A., Trigonometric Series, Cambridge University Press, 1959.

2. ROSENTHAL, H., Biased Coln Convolution Operators, The Altgeld Book, University of Illinols at Urbana, 1976.

3. BONAMI, A., Etude des Coefficients de Fourier des Fonctions de $\mathrm{L}^{\mathrm{P}}$, Ann. Inst. Fourier 20 (1970), 335-402.

4. GARSIA, A., Martingale Inequalities, Seminar Notes on Recent Progress, Math. Lecture Note Series, W.A. Benjamin Inc., 1973.

5. JOHNSON, W., SCHECHTMAN, G. and ZINN, J., Best Constants in Moment Inequalities For Linear Combinations of Independent and Exchangeable Randon Variables, Ann. of Probability 13 (1985), 234-253. 


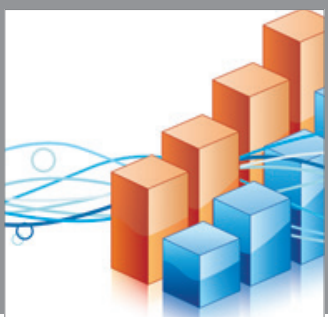

Advances in

Operations Research

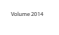

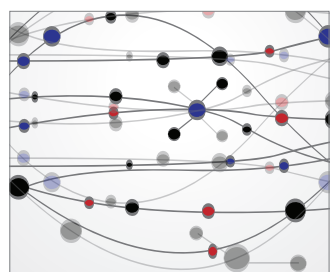

\section{The Scientific} World Journal
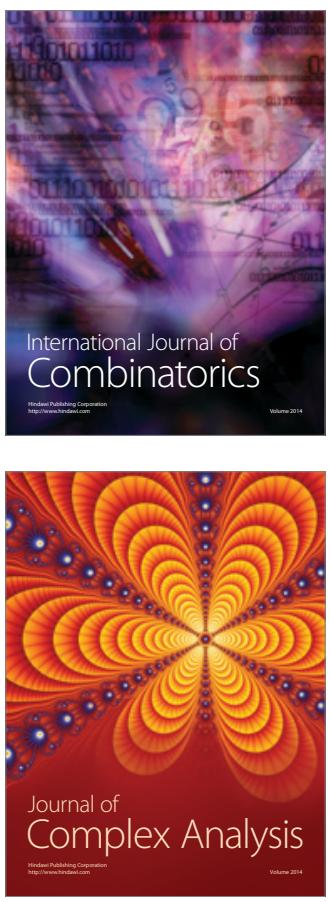

International Journal of

Mathematics and

Mathematical

Sciences
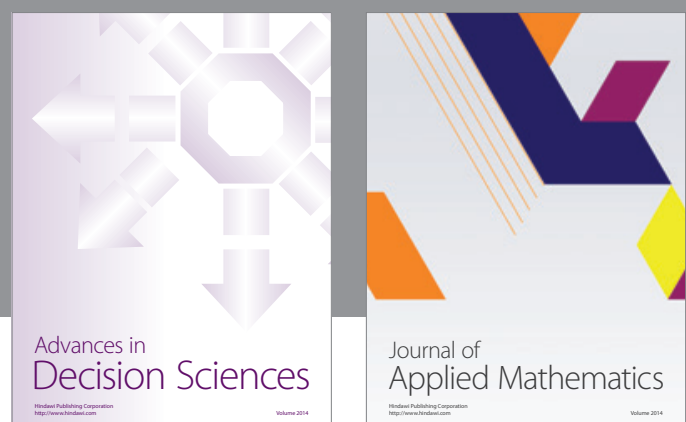

Journal of

Applied Mathematics
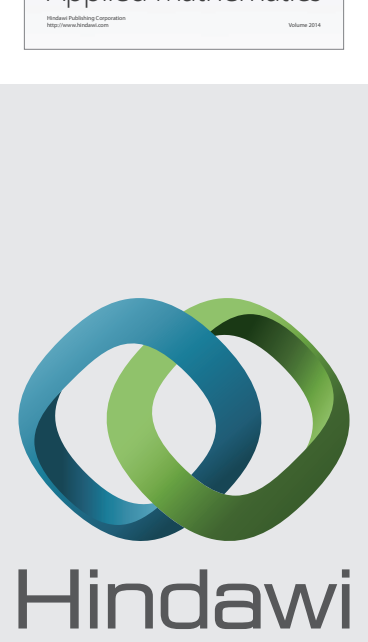

Submit your manuscripts at http://www.hindawi.com
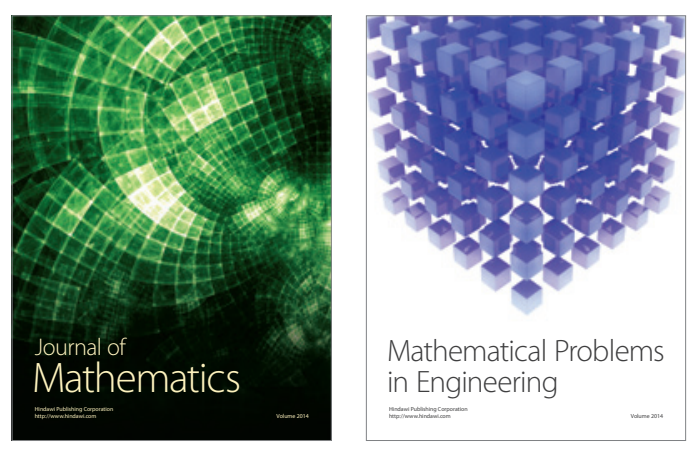

Mathematical Problems in Engineering
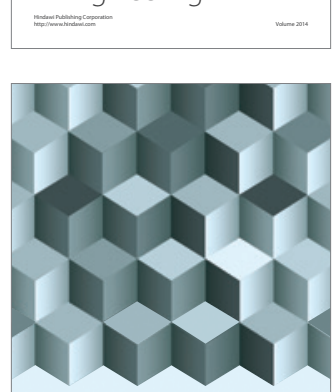

Journal of

Function Spaces
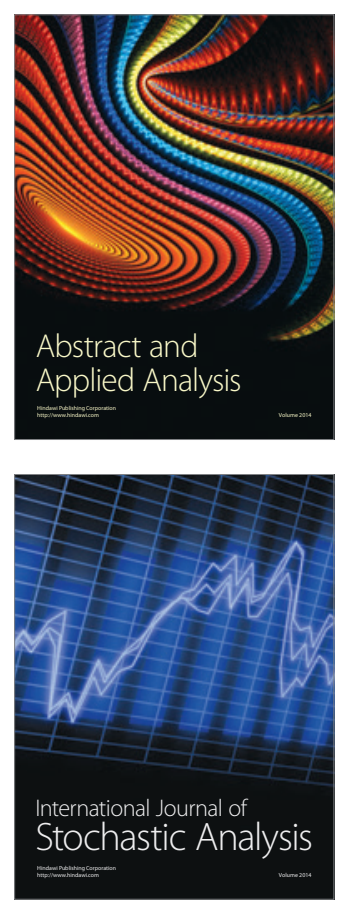

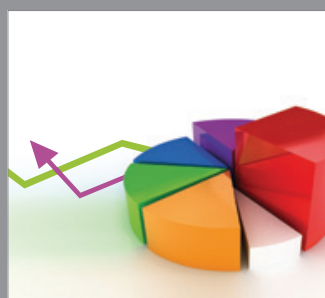

ournal of

Probability and Statistics

Promensencen
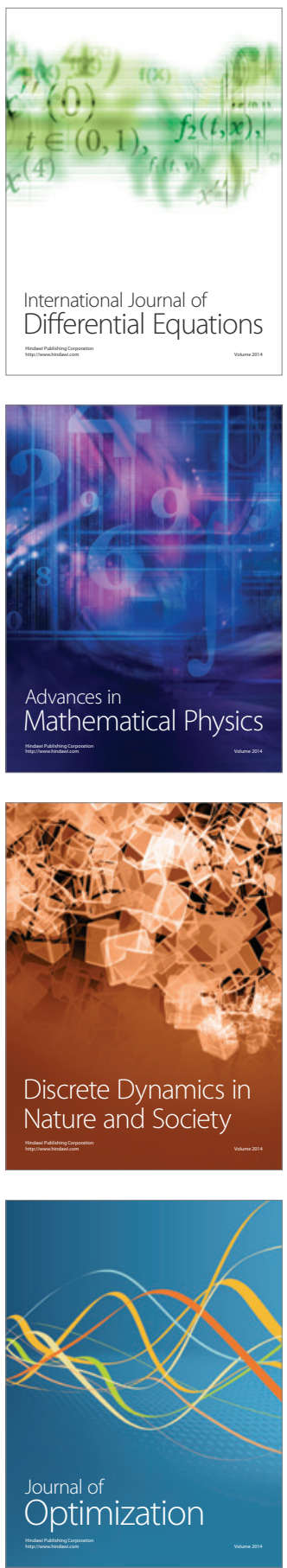